\title{
Correlates of knee bone marrow lesions in younger adults
}

\author{
Benny Antony ${ }^{1}$, Alison Venn ${ }^{1}$, Flavia Cicuttini ${ }^{2}$, Lyn March ${ }^{3}$, Leigh Blizzard ${ }^{1}$, Terence Dwyer ${ }^{4}$, Andrew Halliday ${ }^{5}$, \\ Marita Cross $^{3}$, Graeme Jones $^{1}$ and Changhai Ding ${ }^{1,2^{*}}$
}

\begin{abstract}
Background: Subchondral bone marrow lesions (BMLs) play a key role in the pathogenesis of osteoarthritis (OA) and are associated with pain and structural progression in knee OA. However, little is known about clinical significance and determinants of BMLs of the knee joint in younger adults. We aimed to describe the prevalence and environmental (physical activity), structural (cartilage defects, meniscal lesions) and clinical (pain, stiffness, physical dysfunction) correlates of BMLs in younger adults and to determine whether cholesterol levels measured 5 years prior were associated with current BMLs in young adults.
\end{abstract}

Methods: Subjects broadly representative of the Australian young adult population ( $n=328$, aged 31-41 years, female $48.7 \%$ ) underwent T1- and proton density-weighted fat-suppressed magnetic resonance imaging (MRI) in their dominant knee. BMLs, cartilage defects, meniscal lesions and cartilage volume were measured. Knee pain was assessed by Western Ontario and McMaster Universities Osteoarthritis Index (WOMAC) and physical activity was measured by the International Physical Activity Questionnaire (IPAQ). Cholesterol levels including high-density lipoprotein (HDL) were assessed 5 years prior to MRI.

Results: The overall prevalence of BML was $17 \%$ (grade 1: $10.7 \%$, grade 2: 4.3\%, grade 3: $1.8 \%$ ). BML was positively associated with increasing age and previous knee injury but not body mass index. Moderate physical activity (prevalence ratio (PR):0.93, $95 \%$ Cl: 0.87, 0.99) and HDL cholesterol (PR:0.36, 95 \% Cl: 0.15, 0.87) were negatively associated with BML, while vigorous activity (PR:1.02, $95 \% \mathrm{Cl}: 1.01,1.03$ ) was positively associated with medial tibiofemoral BMLs. BMLs were associated with more severe total WOMAC knee pain ( $>5$ vs $\leq 5$, PR:1.05, $95 \%$ Cl: $1.02,1.09)$ and WOMAC dysfunction (PR:1.75, $95 \%$ Cl: 1.07, 2.89), total knee cartilage defects (PR:2.65, $95 \%$ Cl: $1.47,4.80)$ and total meniscal lesion score (PR:1.92, $95 \%$ Cl: 1.13, 3.28).

Conclusions: BMLs in young adults are associated with knee symptoms and knee structural lesions. Moderate physical activity and HDL cholesterol are beneficially associated with BMLs; in contrast, vigorous physical activity is weakly but positively associated with medial tibiofemoral BMLs.

Keywords: Young adults, Cholesterol, Physical activity, Bone marrow lesions, Cartilage defects, Meniscal lesions

\section{Background}

Osteoarthritis (OA) affects whole joint structure including subchondral bone that results in joint pain and dysfunction. Subchondral bone is rich in blood and nerve supply and there is emerging evidence to suggest that changes in bone

\footnotetext{
* Correspondence: Changhai.Ding@utas.edu.au

${ }^{1}$ Menzies Institute for Medical Research, University of Tasmania, 17 Liverpool Street, Hobart TAS 7000, Australia

${ }^{2}$ Department of Epidemiology and Preventive Medicine, Monash University, 99 Commercial Road, Melbourne, VIC 3004, Australia

Full list of author information is available at the end of the article
}

precede cartilage damage, so that bone rather than cartilage may be the site initiating the significant pathophysiological events in OA $[1,2]$. Subchondral bone marrow lesions (BMLs) play a key role in the pathogenesis of OA [3] and are associated with pain [4]. BMLs can regress and progress over time [5] and BML regression is associated with a decrease in knee pain $[6,7]$. This makes BML an attractive target for treatment of OA [8] and it is being used in clinical trials as an outcome measure $[9,10]$. BMLs in the tibiofemoral joint can predict both structural and clinical changes in knee OA $[11,12]$ and the natural history of 
BMLs has been explored in older adults [13]. Little is known about their determinants and clinical significance in younger population.

The prevalence of vascular disease is high among people with OA [14-16]. These diseases may share some common risk factors such as obesity, high low-density lipoprotein (LDL) levels and elevated total cholesterol [17]. Subchondral bone ischemia may be one of the mechanisms by which vascular pathology contributes to the development of BMLs [18]. Total cholesterol and triglycerides were associated with BMLs in women [19] and high-density lipoprotein (HDL) cholesterol seems to have a protective effect on incidence of BMLs in older adults [18]. Again, these studies are mostly performed in older adults and there are no studies in young adults.

The association of BMLs with physical activity is controversial. The current evidence, mostly in older adults, is mixed with some showing beneficial [20] and some detrimental effects [21, 22]; a recent systematic review found limited evidence overall for an association between physical activity and BMLs [23]. The associations between physical activity and BMLs in young adults have not been explored.

The aims of this study were, therefore, to describe the environmental (physical activity), structural (cartilage defects, meniscal lesions) and clinical (pain, stiffness, physical dysfunction) correlates of BMLs in younger adults and to determine whether cholesterol levels measured 5 years prior were associated with current BMLs in young adults.

\section{Methods}

\section{Study population}

Participants $(n=328$, aged $31-41$ years, female $48.7 \%)$ were broadly representative of the Australian young adult population because they were originally part of the Australian Schools Health and Fitness Survey (ASHFS) of 1985. This survey was conducted in schools Australia-wide and the sampling procedures and methods of data collection are presented elsewhere [24]. The Childhood Determinants of Adult Health (CDAH) study is a 20-year follow-up study of the ASHFS conducted during 2004 to 2006 and included subjects of mean age $31(n=2410$, age range $26-36$ years $)$ who underwent various measurements including their blood cholesterol levels. The current study, known as the CDAH Knee study was conducted during 2008-2010 and participants were originally involved with the $\mathrm{CDAH}$ study [25]. The CDAH participants $(n=764)$ living in Melbourne and Sydney were contacted and invited to participate in the CDAH Knee study. Eligibility criteria were assessed in subjects who agreed to participate $(n=$ 529, response percentage $69 \%$ ). Exclusion criteria included the following: being pregnant; having diseases that may affect knee cartilage such as rheumatoid arthritis; having a contraindication for magnetic resonance imaging (MRI) including claustrophobia. Eighty subjects were excluded, and the remaining 449 subjects were asked to complete a short computer-assisted telephone interview with physical activity and knee pain questionnaires. Injury status to the knee was recorded in response to the question "Have you had a knee injury requiring non-weight-bearing treatment for more than 24 hours or surgery?" They were requested to have a MRI scan of their dominant knee at Epworth Hospital in Melbourne or North Shore Private Hospital in Sydney. Some participants $(n=119)$ did not undergo MRI after enrolling in the study due to the long distance that they would have needed to travel for MRI, work or family commitments, moving interstate, becoming pregnant by the time of MRI, or changing their mind. Two subjects' MRIs were not readable and they were excluded. This study was approved by the Southern Tasmania Health and Medical Human Research Ethics Committee, the Monash University Human Research Ethics Committee and the Northern Sydney and Central Coast Area Human Research Ethics Committee, and all participants provided written informed consent.

\section{Anthropometric measurements}

Weight was measured to the nearest $0.1 \mathrm{~kg}$ in $\mathrm{CDAH}$ study as well as in CDAH Knee study with shoes, socks, and bulky clothing removed. Height was measured to the nearest $0.1 \mathrm{~cm}$ (with shoes and socks removed) using a stadiometer. Waist circumference was measured to the nearest $0.1 \mathrm{~cm}$. Body mass index (BMI) was calculated as kilograms of weight per square metre of height.

\section{Physical activity measurements}

Physical activity status (at work, as part of house and yard work, to get from place to place, in spare time for recreation, exercise and/or sports) in the past 7 days was recorded by requesting the participants in the $\mathrm{CDAH}$ Knee study to complete a short version of the International Physical Activity Questionnaire (IPAQ). Physical activities were calculated to represent minutes per week of vigorous physical activity, moderate physical activity, walking and total physical activity. Vigorous physical activity was recorded using the question: "think about all the vigorous activities which take hard physical effort that you did in the last 7 days. Vigorous activities make you breathe much harder than normal and may include heavy lifting, digging, aerobics, or fast bicycling. Think only about those physical activities that you did for at least 10 minutes at a time". Regular participation is a key concept included in current public health guidelines for physical activity [26]. Therefore, both the total volume and the number of day/sessions are included in 
the IPAQ analysis algorithms. The IPAQ demonstrates very good levels of repeatability and fair to moderate validity when compared to accelerometer data [27].

\section{Knee symptom measures}

Knee symptoms during the past 30 days were assessed using the Western Ontario and McMaster Universities osteoarthritis index (WOMAC) scale in CDAH Knee study. Subjects were asked about the knee pain, stiffness and physical dysfunction status during computer-assisted telephone interview. Each question was graded on a scale of $0-9$, where 0 indicated no symptoms and 9 indicated the maximum intensity of the symptoms. The WOMAC is an established scale for OA research and is also validated for responsiveness of knee complaints in young population without OA [28]. Total WOMAC scores were calculated by adding the scores of 5 subscales in WOMAC knee pain, 2 subscales in WOMAC stiffness and 17 subscales in WOMAC dysfunction. Presence of any pain, stiffness and dysfunction was defined as any score $\geq 1$. Total WOMAC knee pain was also categorised into two groups based on total WOMAC score of $>5$ and $\leq 5$.

\section{Cholesterol measures}

Venous blood samples were collected from the antecubital vein after a 12-hour fast in $\mathrm{CDAH}$ study approximately 5 years prior to CDAH Knee study. Serum total cholesterol and HDL cholesterol concentrations were determined enzymatically (Olympus AU5400 automated analyzer, Olympus Optical, Tokyo, Japan) and the Lipid Research Clinic procedures were followed. LDL cholesterol concentration was calculated using the Friedewald formula [29].

\section{Blood pressure measurements}

Blood pressure was recorded while seated, after resting quietly for 5 minutes, and cuff-size (small, medium, large) was determined using arm circumference. Systolic and diastolic blood pressure measurements were taken from the right brachial artery using the Omron HEM907 digital automatic monitor (Omron Healthcare Co, Ltd, Kyoto, Japan). The mean of three readings with a 1minute interval between each was recorded.

\section{Insulin resistance}

Fasting plasma glucose levels were measured by the Olympus AU5400 automated analyser (Olympus, Southend-onSea, UK). Fasting plasma insulin was measured by a microparticle enzyme immunoassay kit (AxSYM; Abbot Laboratories, Abbot Park, IL, USA) and by electrochemiluminescence immunoassay (Elecsys Modular Analytics E170; Roche Diagnostics, Mannheim, Switzerland) with interassay standardization. Insulin sensitivity was estimated by the homeostasis model assessment (HOMA) method. HOMA is determined from fasting plasma insulin and glucose where: HOMA = fasting insulin $(\mu \mathrm{U} / \mathrm{mL}) \times$ fasting glucose $(\mathrm{mmol} / \mathrm{L}) / 22.5$.

\section{MRI measurements}

Participants had an MRI scan of their dominant knee in CDAH Knee study. MRI scans were obtained from two hospitals, which used the same type of machine (General Electric Medical Systems, Milwaukee, WI, USA). Knees were imaged in the sagittal plane on a $1.5 \mathrm{~T}$ whole body magnetic resonance unit with use of a commercial transmit-receive extremity coil. The following image sequence was used: (1) T1-weighted fat saturation threedimensional spoiled gradient recall acquisition in the steady state; flip angle 55 degrees; repetition time 58 msecs; echo time $12 \mathrm{msec}$; field of view $16 \mathrm{~cm}$; 60 partitions; $512 \times 512$ matrix; acquisition time $11 \mathrm{~min} 56 \mathrm{sec}$; one acquisition. Sagittal images were obtained at a partition thickness of $1.5 \mathrm{~mm}$ and an in-plane resolution of $0.31 \times 0.31$ (512 $\times 512$ pixels). (2) Proton densityweighted fat saturated two-dimensional fast spin-echo coronal images at a partition thickness of $3.3 \mathrm{~mm}$ and an in-plane resolution of $0.31 \times 0.31(512 \times 512$ pixels $)$.

BMLs were measured using the coronal proton densityweighted images and was marked as the increased signal intensity area in the subchondral bone adjacent to the osteochondral junction. BMLs were scored in medial and lateral compartment of tibia and femur using an ordinal scoring system which we used previously [11]. Subjects with no BMLs were scored as grade 0 and then the subjects with BMLs were graded according to the percentage of area of occupancy of BML in each compartment: grade 1: $\leq 25 \%$ of area; grade 2: $>25 \%$ to $<50 \%$; grade 3 : $>50 \%$.

Cartilage defects were measured using both proton density coronal images and T1-weighted sagittal images. Cartilage defects were graded in an ordinal scale as we published before [30]. Grade 0 indicated a normal cartilage, grade 1 indicated focal blistering and low (T1-weighted) or high (proton density-weighted) signal intensity area with intact surface/bottom. Grade 2 indicated a loss of thickness of less than $50 \%$ on surface/bottom of the cartilage. Grade 3 represented a deep ulceration with loss of thickness $>50 \%$ and grade 4 indicated a full-thickness chondral wear with exposure of subchondral bone. A prevalent cartilage defect was defined as a cartilage defect score of $\geq 2$ at any site within that compartment.

Meniscal tear was graded in medial and lateral menisci separately based on a combined Whole-Organ Magnetic Resonance Imaging Score (WORMS) scoring system [31] from grade 0 to 2 using proton density-weighted coronal and T1-weighted sagittal images. Grade 0 was a fully normal intact meniscus. Grade 1 indicated a nondisplaced tear (scored as grade 1 and 2 by WORMS). 
Grade 2 indicated a displaced tear or maceration (scored as grade 3 and 4 by WORMS).

Meniscal extrusion was recorded on the medial and lateral menisci and was graded from grade 0 to 2 based on the proton density-weighted coronal images as published before [32]. Grade 0 was an intact meniscus without any degree of extrusion. Grade 1 indicated a partially displaced meniscus with respect to tibia and grade 2 represented a complete displaced meniscus. Meniscal lesions were defined as any meniscal tear or meniscal extrusion in the knee.

\section{Statistical analyses}

Mean and standard deviation or the percentages of the subjects were used for calculating the characteristics of the participants. $t$ test or chi-square test was used to compare the characteristics of the participants based on the presence or absence of BML. Univariable and multivariable $\log$ binomial regression was used to estimate prevalence ratio (PR) of the associations between $\mathrm{BML}$ (dependent variable) and knee symptoms, structural pathologies, physical activity and cholesterol (independent variable) before and after adjustment for potential confounders. If the log binomial model failed to converge, PR was estimated using a Poisson distribution and robust standard errors. Age, gender, BMI, previous knee injury and/or duration of follow-up (for cholesterol analyses only) were examined as potential confounders based on the significant associations with BMLs or our previous literature suggesting that these are important covariates. All statistical analyses were performed on IBM SPSS 19 for Mac (IBM Corp., Armonk, NY, USA).

\section{Results}

The prevalence of BML in the knee joint was $17 \%$ (grade 1: $10.7 \%$, grade 2: $4.3 \%$, grade $3: 1.8 \%$ ). The baseline characteristics of the participants based on the presence or absence of BMLs are presented in Table 1. Participants with BML were similar in terms of gender distribution, BMI, total physical activity and total and LDL cholesterol when compared with participants without BMLs. However, participants with BMLs had lower moderate physical activity, higher proportion with cartilage defects, meniscal lesions, and WOMAC pain, and dysfunction. HDL cholesterol was lower in participants with BMLs compared to those without BMLs.

Cross-sectional associations between demographic factors, physical activity and BMLs are shown in Table 2. Age was associated with BML in univariable and multivariable analysis. Gender, BMI, and previous knee injury were not significantly associated with BML; however, previous knee injury was associated with medial tibiofemoral BMLs (PR: $2.20,95 \%$ confidence interval $(\mathrm{CI}): 1.03,4.71)$ in multivariable analysis. Total physical activity was not associated with
Table 1 Baseline characteristics of the participants based on their bone marrow lesion status

\begin{tabular}{llll}
\hline & No BML & BML (Yes) & $P$ value \\
& $(n=272)$ & $(n=55)$ & \\
\hline Age (years) & $35.2(2.7)$ & $36.0(2.6)$ & 0.053 \\
Sex (male, \%) & 52 & 58 & 0.239 \\
BMI (kg/m²) & $25.7(4.3)$ & $25.6(4.0)$ & 0.864 \\
Knee injury (\%) & 16 & 24 & 0.113 \\
Total physical activity (min/week) & $927.4(1308.9)$ & $932.6(1354.1)$ & 0.979 \\
Total vigorous PA (min/week) & $205.2(446.6)$ & $349.0(987.9)$ & 0.094 \\
Total moderate PA (min/week) & $277.3(621.6)$ & $122.7(189.2)$ & 0.001 \\
Cartilage defects (\%) & 4.4 & 16.4 & 0.003 \\
Total meniscal extrusion (\%) & 3.3 & 11.1 & 0.024 \\
Total meniscal tear (\%) & 12 & 22 & 0.051 \\
Total meniscal lesions (\%) & 13.6 & 27.3 & 0.013 \\
WOMAC pain (yes, \%) & 34 & 45 & 0.084 \\
WOMAC stiffness (yes, \%) & 31 & 41 & 0.075 \\
WOMAC dysfunction (yes, \%) & 39 & 56 & 0.018 \\
WOMAC pain (>5 vs $\leq 5, \%)$ & 11 & 22 & 0.032 \\
HDL cholesterol (fasting, mmol/L) & $1.45(0.34)$ & $1.34(0.28)$ & 0.019 \\
LDL cholesterol (fasting, mmol/L) & $2.90(0.80)$ & $2.87(0.69)$ & 0.727 \\
Total cholesterol (fasting, mmol/L) & $4.86(0.93)$ & $4.69(0.74)$ & 0.145 \\
\hline Two-tail & &
\end{tabular}

Two-tailed $t$ tests used for differences between means; $\mathrm{X} 2$ test used for proportions (percentages)

Mean (SD) except for percentages

$B M L$ bone marrow lesion, $B M I$ body mass index, $P A$ physical activity, WOMAC Western Ontario and McMaster Universities osteoarthritis index, HDL high-density lipoprotein, $L D L$ low-density lipoprotein

BML in the knee, but moderate physical activity was protectively associated with BML before and after adjustment for age, gender, BMI and knee injury. Vigorous physical activity showed a weak but deleterious association with medial tibiofemoral BMLs (PR: 1.02, 95 \% CI: 1.01, 1.03) and the association with any knee BMLs was of borderline significance. These associations remained significant after further adjustment for cartilage defects and meniscal lesions.

HDL cholesterol measured approximately 5 years prior was negatively associated with BML in univariable and multivariable analysis (Table 3). This association remained significant after further adjustment for HOMA, systolic blood pressure and waist circumference measured 5 years prior. Figure 1 shows the prevalence of BMLs based on quartiles of HDL cholesterol. Total cholesterol or LDL cholesterol was not significantly associated with BML of the knee after adjustment for age, gender, BMI, duration of follow-up and knee injury (Table 3).

Figure 2 shows the prevalence of BMLs in each pain category based on the grouping of total WOMAC pain $(0, \geq 1$ to $5,>5)$. BMLs in the knee was not significantly associated with any total WOMAC knee pain $(\geq 1 \mathrm{vs}=0)$; however, when we categorised total WOMAC knee pain 
Table 2 Associations of demographic factors and physical activity with bone marrow lesions in young adults

\begin{tabular}{|c|c|c|}
\hline & Univariable PR (95 \% Cl) & Multivariable ${ }^{a}$ PR $(95 \%$ Cl) \\
\hline Age (per year) & $1.09(1.00,1.20)$ & $1.10(1.00,1.20)$ \\
\hline Sex (females vs males) & $1.24(0.76,2.02)$ & $1.26(0.76,2.08)$ \\
\hline BMI $\left(\right.$ per $\left.\mathrm{kg} / \mathrm{m}^{2}\right)$ & $1.00(0.94,1.05)$ & $0.99(0.93,1.05)$ \\
\hline Knee injury (yes vs no) & $1.50(0.87,2.60)$ & $1.49(0.86,2.58)$ \\
\hline Total physical activity (per hour/week) & $1.00(0.99,1.01)$ & $1.00(0.99,1.01)$ \\
\hline Total vigorous PA (per hour/week) & $1.01(1.00,1.02)$ & $1.01(0.99,1.02)$ \\
\hline Total moderate PA (per hour/week) & $0.94(0.88,0.99)$ & $0.93(0.87,0.99)$ \\
\hline
\end{tabular}

Bold denotes statistical significance at $P<0.05$

$P R$ prevalence ratio, $95 \% \mathrm{Cl} 95 \%$ confidence interval, $B M I$ body mass index, $P A$ physical activity

${ }^{\mathrm{a} A d j u s t e d}$ for age, sex, BMI, and knee injury

as $>5$ vs $\leq 5$ for a severity analysis, there was a significant association with BMLs before and after adjustment for covariates (Table 4). However, the association became of borderline significance $(P=0.072)$ after further adjustment for cartilage defects, but was independent of meniscal lesions.

The prevalence of knee pain in grade 1, 2 and 3 BMLs were $37 \%, 57 \%$ and $67 \%$, respectively. The prevalence ratios of grade 1 and grade 2 or 3 BMLs (grade 0 as the reference) with WOMAC knee pain, stiffness and dysfunction are presented in Table S1 in Additional file 1. Higher grades of BMLs (grade 2 and 3 combined) were significantly associated with higher prevalence of any WOMAC pain, WOMAC pain $>5$ and physical dysfunction (Table $\mathrm{S} 1$ in Additional file 1). There was a dose-response relationship between BML grades and prevalence of WOMAC knee pain $>5$ (Figure S1 in Additional file 1). Presence of BML was also associated with any WOMAC dysfunction in univariable and multivariable analyses (Table 4). These associations remained largely unchanged after further adjustment for cartilage defects and meniscal lesions.

As shown in Table 4, BMLs were associated with other knee structural abnormalities including total knee cartilage defects and total meniscal extrusion, as well as total meniscal lesions defined as any tear or extrusion of the menisci. BMLs were not significantly associated with cartilage volume or bone area in these young adults (data not shown).

\section{Discussion}

This is the first study exploring the correlates of knee BMLs in a population-based sample of young adults. Knee BMLs were surprisingly commonly being found in $17 \%$ of participants. They were associated with increasing age, previous knee injury, increased knee symptoms and structural abnormalities such as meniscal lesions and cartilage defects. Furthermore, moderate physical activity and higher HDL cholesterol were associated with decreased while vigorous activity was weakly associated with increased BML, suggesting that BMLs in young adults are modifiable.

BMLs are commonly found in knee OA patients and are also present in non-OA populations. Prevalence of BMLs in this young population-based cohort was $17 \%$, which is comparable with prevalence reported previously in an older but non-symptomatic or non-OA population [33-35]. Similarly, not all participants with BMLs were symptomatic in our study. Pathology of BMLs are complex and BML zone mainly consisted of normal tissue (53\% of the area was fatty marrow, $16 \%$ was intact trabeculae, and $2 \%$ was blood vessels) and a smaller proportion of several abnormalities (bone marrow necrosis [11 \% of area], abnormal [necrotic or remodelled] trabeculae [8 \%], bone marrow fibrosis [4\%], bone marrow oedema [4\%], and bone marrow bleeding [2\%]) [36]. Subchondral bone is rich in blood supply and it is possible that the systemic and local factors can play a role in the BML pathology. We found that age was associated

Table 3 Association of cholesterol measured approximately 5 years prior with bone marrow lesion

\begin{tabular}{lll}
\hline & Univariable PR $(95 \% \mathrm{Cl})$ & Multivariable $^{\mathrm{a}} \mathrm{PR}(95 \% \mathrm{Cl})$ \\
\hline $\mathrm{HDL}$ cholesterol (per mmol/L) & $\mathbf{0 . 4 2}(\mathbf{0 . 2 0 , 0 . 8 8 )}$ & $\mathbf{0 . 3 6}(\mathbf{0 . 1 5}, \mathbf{0 . 8 7})$ \\
$\mathrm{LDL}$ cholesterol (per mmol/L) & $0.95(0.71,1.27)$ & $0.94(0.69,1.27)$ \\
Total cholesterol (per mmol/L) & $0.83(0.65,1.07)$ & $0.84(0.66,1.06)$ \\
\hline
\end{tabular}

Bold denotes statistical significance at $P<0.05$

$P R$ prevalence ratio, $95 \% \mathrm{Cl} 95 \%$ confidence interval, $H D L$ high-density lipoprotein, $L D L$ low-density lipoprotein

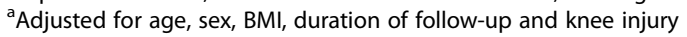




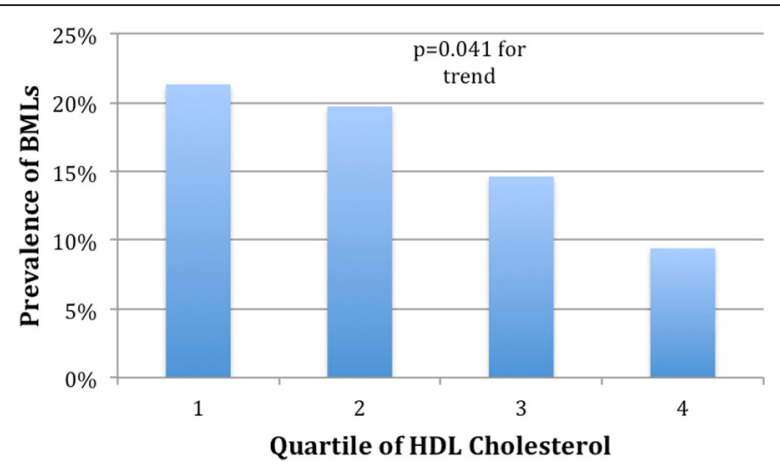

Fig. 1 Association between prevalence of any bone marrow lesion and quartile of high-density lipoprotein cholesterol measured approximately 5 years prior. $P$ value from multivariable log binomial regression after adjusting for age, sex, body mass index, knee injury and duration of follow-up. BMLs bone marrow lesions, HDL high-density lipoprotein

with increased risk of BMLs, which is consistent with a finding in a non-OA population cohort [37]. Injury was associated with medial tibiofemoral BMLs, which is in line with the finding observed in middle-aged and older adults [38]. However, BMI was not significantly associated with BMLs in this young cohort. Similar results were also observed in healthy middle-aged and older adults [37, 39]. In contrast, a systematic review has suggested a moderate level of evidence for the association between obesity measures and BMLs [40].

The current evidence of the association of physical activity with BMLs are mostly in older adults and suggests beneficial [20], detrimental [21, 22, 41] or no effect [37, 42]. Racunica et al. reported that non-vigorous activity (less vigorous activity and walking) was negatively associated with BMLs [20] in healthy older adults; in contrast, we reported that strenuous physical activity independently predicted an increase in BML size in middle-aged adults [5]. Similarly, we found that doing $\geq 10000$ steps per day was

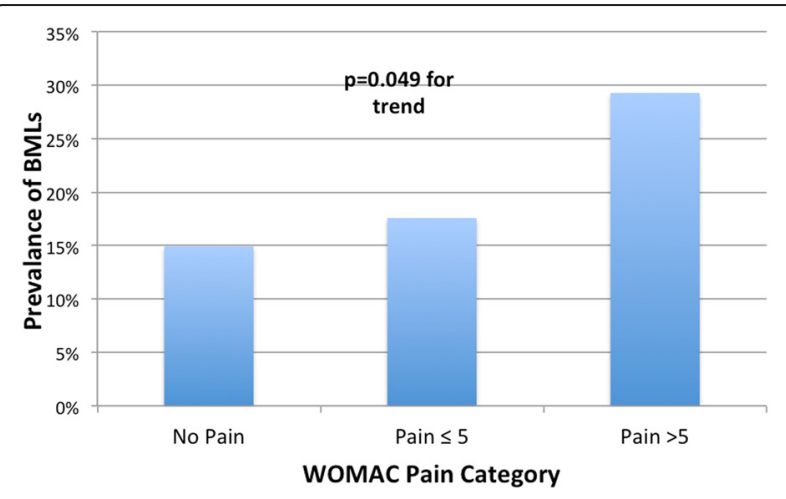

Fig. 2 Association between prevalence of any bone marrow lesion and category of total knee pain (0-45). $P$ value from multivariable log binomial regression after adjusting for age, sex, body mass index and knee injury. BMLs bone marrow lesions, WOMAC Western Ontario and McMaster Universities osteoarthritis index associated with an increase in BML in older adults [22]. Asymptomatic middle-aged individuals from the "Osteoarthritis Initiative" incidence cohort had more knee BMLs in those who were more physically active, independent of confounders [21]. We found in this young population that although total physical activity was not associated with BMLs, moderate physical activity was associated with reduced BMLs in the whole knee but vigorous physical activity was weakly associated with increased BMLs in medial tibiofemoral compartment. These associations were independent of cartilage defects and meniscal lesion. These findings suggest that different levels of physical activity may have different influences on subchondral bone health in young adults.

Vascular pathology is proposed in the pathophysiology of OA. Increased popliteal artery wall thickness was associated with reduced medial tibial cartilage volume, increased rate of cartilage volume loss and a trend for BML worsening over 2 years [43]. Total cholesterol and triglycerides were associated with increased BMLs in women [19]. In our study, we found that although total cholesterol and LDL measured 5 years prior were not associated with BMLs, HDL cholesterol measured 5 years prior was significantly associated with reduced BMLs. This association was independent of other metabolic syndrome measurements such as blood pressure, waist circumference and HOMA. This is consistent with our previous longitudinal findings in older adults where HDL cholesterol was associated with BML resolution and had a protective effect on incidence of BMLs [18]. HDL cholesterol is considered to be protective against vascular pathology through cholesterol transport, antiinflammatory, and antioxidant effects, and therefore may help to reduce BML development and progression [44].

Subchondral bone is richly innervated with nociceptive pain fibres [45]. Thus, subchondral bone could be a source of knee pain. There is increasing evidence to suggest that BMLs are associated with knee pain [4, 46-48] in older adults or knee OA patients, though some studies did not establish this association [49, 50]. A systematic review suggested that there was moderate evidence for the association between BMLs and knee pain [51]. We found that BMLs were associated with more severe knee pain when the total WOMAC pain was categorized at 5 in young adults, though this association was in part dependent of cartilage defects. Higher grades of BMLs were independently associated with greater knee pain, suggesting that BMLs in young adults would also be a source of knee pain.

Structurally, BMLs appear to be sclerotic compared with unaffected regions from the same individual based on the increased bone volume fraction and increased trabecular thickness [52, 53]. The mineral density in these lesions, however, is reduced and may render this area to be 
Table 4 Association of bone marrow lesions with knee symptoms and structural abnormalities

\begin{tabular}{|c|c|c|}
\hline & Univariable PR (95% Cl) & Multivariable ${ }^{a}$ PR $(95 \%$ Cl) \\
\hline \multicolumn{3}{|l|}{ Symptoms } \\
\hline WOMAC pain (any vs no) & $1.46(0.90,2.35)$ & $1.41(0.86,2.33)$ \\
\hline WOMAC stiffness (any vs no) & $1.49(0.92,2.40)$ & $1.48(0.90,2.45)$ \\
\hline WOMAC dysfunction (any vs no) & $1.75(1.07,2.84)$ & $1.75(1.07,2.89)$ \\
\hline WOMAC pain (>5 vs $\leq 5)$ & $1.87(1.08,3.24)$ & $1.80(1.03,3.14)$ \\
\hline \multicolumn{3}{|l|}{ Structural abnormalities } \\
\hline Cartilage defects (any vs no) & $2.85(1.63,4.50)$ & $2.54(1.49,4.34)$ \\
\hline Meniscal extrusion (any vs no) & $2.59(1.32,5.08)$ & $2.50(1.29,4.82)$ \\
\hline Meniscal tear (any vs no) & $1.75(1.00,3.05)$ & $1.63(0.93,2.86)$ \\
\hline Meniscal lesion (any vs no) & $1.98(1.19,3.32)$ & $1.92(1.13,3.28)$ \\
\hline
\end{tabular}

Bold denotes statistical significance at $P<0.05$

$P R$ prevalence ratio, $95 \% \mathrm{Cl} 95 \%$ confidence interval, WOMAC Western Ontario and McMaster Universities osteoarthritis index

${ }^{\mathrm{a}}$ Adjusted for age, sex, BMI, and knee injury

mechanically compromised, and thus susceptible to attrition [52]. Therefore, it is highly likely that BMLs can have a local mechanical effect on the knee joint. It has been reported that BMLs can predict site-specific cartilage pathology including cartilage defect progression and cartilage volume loss in older [11, 54] and middle-aged [34] adults. Similarly, BMLs are associated with meniscal pathology [55] and meniscal pathology increases the risk for the incidence and progression of BMLs in older adults [56]. This study is the first to report that in young adults, BMLs were associated with meniscal lesions and cartilage defects. We did not find any significant association with knee cartilage volume or bone area, which concurs with the results from a study in healthy middle-aged women [33], indicating that BMLs may be associated with earlier structural changes such as cartilage defects rather than later structural changes such as reduced cartilage volume in young populations.

A strength of our study was the use of a populationbased sample in young adults. This study has several potential limitations. The response rate was low with only $43 \%$ of the persons invited to participate having MRI performed. Reassuringly, there were no significant differences in age, sex, BMI, and knee injury between those with and without MRI scans, or between subjects included in this study and the remainder of the original cohort, which suggests there was no major selection bias introduced. We did not record the severity of injury in this study and therefore cannot assess the effect of injury severity on BMLs. Cholesterol measures were assessed 5 years prior and we did not have MRI measurements at that time, so cannot examine if cholesterol was associated with change in BMLs over time. We did not record separate sports activities that could have contributed to the vigorous physical activity. The strength of the association of physical activity with BML was very low and the results need to be interpreted cautiously. Most of these findings were based on cross-sectional data and therefore the causal pathways cannot be ascertained.

\section{Conclusions}

In conclusion, BMLs in young adults are associated with knee symptoms, a history of knee injury and other knee structural lesions. Moderate physical activity and HDL cholesterol are beneficially associated with BMLs, in contrast, vigorous physical activity is weakly but positively associated with medial tibiofemoral BMLs. These suggest that BMLs are modifiable in young adults.

\section{Additional file}

Additional file 1: Table S1. Associations between different grades of bone marrow lesions and knee symptoms. Figure S1. Association between different grades of bone marrow lesions and prevalence of WOMAC knee pain >5. (DOCX $25 \mathrm{~kb}$ )

\section{Abbreviations}

ASHFS: Australian Schools Health and Fitness Survey; BMI: Body mass index; BMLs: Bone marrow lesions; CDAH: Childhood Determinants of Adult Health; $\mathrm{Cl}$ : confidence interval; HDL: High-density lipoprotein; HOMA: Homeostasis model assessment; IPAQ: International Physical Activity Questionnaire; LDL: Low-density lipoprotein; MRI: Magnetic resonance imaging; OA: Osteoarthritis; PR: Prevalence ratio; WOMAC: Western Ontario and McMaster Universities Osteoarthritis Index; WORMS: Whole-Organ Magnetic Resonance Imaging Score.

\section{Competing interests}

All authors have completed the Competing Interest form (available on request from the corresponding author) and declare: no support from any organisation for the submitted work; no financial relationships with any organisations that might have an interest in the submitted work in the previous 3 years; no other relationships or activities that could appear to have influenced the submitted work.

\section{Authors' contributions}

BA had full access to all of the data in the study and takes responsibility for the integrity of the data and the accuracy of the data analysis. BA was responsible for data management and cleaning, carried out analysis and interpretation of data, prepared the initial manuscript draft, and completed 
manuscript revisions. AV, TD, LM and FC designed and carried out the study planning, and critically revised the manuscript. LB participated in analysis and interpretation of data, and critically revised the manuscript. MC and $\mathrm{AH}$ participated in collection of data, and critically revised the manuscript. GJ designed and carried out the study planning, participated in the interpretation of data, and critically revised the manuscript. CD designed and carried out the study planning, participated in analysis and interpretation of the data, assisted with the initial manuscript draft, and critically revised the manuscript. All authors read and approved the manuscript.

\section{Acknowledgements}

Special thanks go to the subjects who made this study possible. The roles of Liz O'Loughlin and Judy Hankin in collecting the data, Marita Dalton in managing the database, Rob Warren in processing the MR images and Petr Otahal in statistical analyses are gratefully acknowledged. C. Ding is a recipient of Australian Research Council Future Fellowship, G. Jones is a recipient of a NHMRC Practitioner Fellowship, A. Venn is a recipient of a NHMRC Senior Research Fellowship, and L. Blizzard is a recipient of a NHMRC Career Development Fellowship. National Health and Medical Research Council of Australia funded this study and the funders had no role in study design, data collection and analysis, decision to publish, or preparation of the manuscript.

\section{Funding}

National Health and Medical Research Council of Australia.

\section{Author details}

'Menzies Institute for Medical Research, University of Tasmania, 17 Liverpool Street, Hobart TAS 7000, Australia. ${ }^{2}$ Department of Epidemiology and Preventive Medicine, Monash University, 99 Commercial Road, Melbourne, VIC 3004, Australia. ${ }^{3}$ Institute of Bone and Joint Research, University of Sydney, Sydney, NSW 2006, Australia. ${ }^{4}$ Murdoch Children's Research Institute, 50 Flemington Road, Melbourne, VIC 3052, Australia. ${ }^{5}$ Department of Radiology, Royal Hobart Hospital, 48 Liverpool Street, Hobart, TAS 7000, Australia.

\section{Received: 8 November 2015 Accepted: 19 January 2016} Published online: 26 January 2016

\section{References}

1. Bailey AJ, Buckland-Wright C, Metz D. The role of bone in osteoarthritis. Age Ageing. 2001;30:374-8.

2. Burr DB. Increased biological activity of subchondral mineralized tissues underlies the progressive deterioration of articular cartilage in osteoarthritis. J Rheumatol. 2005;32:1156-8. discussion 1158-1159.

3. Felson DT, McLaughlin S, Goggins J, LaValley MP, Gale ME, Totterman S, et al. Bone marrow edema and its relation to progression of knee osteoarthritis. Ann Intern Med. 2003;139:330-6.

4. Felson DT, Chaisson CE, Hill CL, Totterman SM, Gale ME, Skinner KM, et al. The association of bone marrow lesions with pain in knee osteoarthritis. Ann Intern Med. 2001;134:541-9.

5. Foong YC, Khan HI, Blizzard L, Ding C, Cicuttini F, Jones G, et al. The clinical significance, natural history and predictors of bone marrow lesion change over eight years. Arthritis Res Ther. 2014;16:R149.

6. Driban JB, Price L, Lo GH, Pang J, Hunter DJ, Miller E, et al. Evaluation of bone marrow lesion volume as a knee osteoarthritis biomarker-longitudinal relationships with pain and structural changes: data from the Osteoarthritis Initiative. Arthritis Res Ther. 2013;15:R112.

7. Zhang Y, Nevitt M, Niu J, Lewis C, Torner J, Guermazi A, et al. Fluctuation of knee pain and changes in bone marrow lesions, effusions, and synovitis on magnetic resonance imaging. Arthritis Rheum. 2011;63:691-9.

8. Davies-Tuck ML, Wluka AE, Wang Y, English DR, Giles GG, Cicuttini F. The natural history of bone marrow lesions in community-based adults with no clinical knee osteoarthritis. Ann Rheum Dis. 2009;68:904-8.

9. Laslett LL, Dore DA, Quinn SJ, Boon P, Ryan E, Winzenberg TM, et al. Zoledronic acid reduces knee pain and bone marrow lesions over 1 year: a randomised controlled trial. Ann Rheum Dis. 2012;71:1322-8.

10. Callaghan MJ, Parkes MJ, Hutchinson CE, Gait AD, Forsythe LM, Marjanovic EJ et al. A randomised trial of a brace for patellofemoral osteoarthritis targeting knee pain and bone marrow lesions. Ann Rheum Dis. 2015;74:1164-70.

11. Dore D, Martens A, Quinn S, Ding C, Winzenberg T, Zhai G, et al. Bone marrow lesions predict site-specific cartilage defect development and volume loss: a prospective study in older adults. Arthritis Res Ther. 2010;12:R222.

12. Hunter DJ, Zhang Y, Niu J, Goggins J, Amin S, LaValley MP, et al. Increase in bone marrow lesions associated with cartilage loss: a longitudinal magnetic resonance imaging study of knee osteoarthritis. Arthritis Rheum. 2006:54:1529-35.

13. Dore D, Quinn S, Ding C, Winzenberg T, Zhai G, Cicuttini F, et al. Natural history and clinical significance of MRI-detected bone marrow lesions at the knee: a prospective study in community dwelling older adults. Arthritis Res Ther. 2010;12:R223.

14. Jonsson H, Helgadottir GP, Aspelund T, Eiriksdottir G, Sigurdsson S, Ingvarsson T, et al. Hand osteoarthritis in older women is associated with carotid and coronary atherosclerosis: the AGES Reykjavik study. Ann Rheum Dis. 2009;68:1696-700.

15. Rahman MM, Kopec JA, Anis AH, Cibere J, Goldsmith CH. Risk of cardiovascular disease in patients with osteoarthritis: a prospective longitudinal study. Arthritis Care Res (Hoboken). 2013;65:1951-8.

16. Rahman MM, Kopec JA, Cibere J, Goldsmith $\mathrm{CH}$, Anis $\mathrm{AH}$. The relationship between osteoarthritis and cardiovascular disease in a population health survey: a cross-sectional study. BMJ Open. 2013; 3. doi: 10.1136/bmjopen-2013-002624.

17. Findlay DM. Vascular pathology and osteoarthritis. Rheumatology (Oxford). 2007:46:1763-8

18. Dore D, de Hoog J, Giles G, Ding C, Cicuttini F, Jones G. A longitudinal study of the association between dietary factors, serum lipids, and bone marrow lesions of the knee. Arthritis Res Ther. 2012;14:R13.

19. Davies-Tuck ML, Hanna F, Davis SR, Bell RJ, Davison SL, Wluka AE, et al. Total cholesterol and triglycerides are associated with the development of new bone marrow lesions in asymptomatic middle-aged women - a prospective cohort study. Arthritis Res Ther. 2009;11:R181.

20. Racunica TL, Teichtahl AJ, Wang Y, Wluka AE, English DR, Giles GG, et al. Effect of physical activity on articular knee joint structures in communitybased adults. Arthritis Rheum. 2007:57:1261-8.

21. Stehling C, Lane NE, Nevitt MC, Lynch J, McCulloch CE, Link TM. Subjects with higher physical activity levels have more severe focal knee lesions diagnosed with 3T MRI: analysis of a non-symptomatic cohort of the osteoarthritis initiative. Osteoarthritis Cartilage. 2010;18:776-86.

22. Dore DA, Winzenberg TM, Ding C, Otahal P, Pelletier JP, Martel-Pelletier J, et al. The association between objectively measured physical activity and knee structural change using MRI. Ann Rheum Dis. 2013;72:1170-5.

23. Lim YZ, Wang Y, Wluka AE, Davies-Tuck ML, Teichtahl A, Urquhart DM, et al. Are biomechanical factors, meniscal pathology, and physical activity risk factors for bone marrow lesions at the knee? A systematic review. Semin Arthritis Rheum. 2013:43:187-94.

24. Dwyer T, Gibbons LE. The Australian Schools Health and Fitness Survey. Physical fitness related to blood pressure but not lipoproteins. Circulation. 1994:89:1539-44.

25. Antony B, Jones G, Venn A, Cicuttini F, March L, Blizzard L, et al. Association between childhood overweight measures and adulthood knee pain, stiffness and dysfunction: a 25-year cohort study. Ann Rheum Dis. 2015;74:711-7.

26. Pate RR, Pratt M, Blair SN, Haskell WL, Macera CA, Bouchard C, et al. Physical activity and public health. A recommendation from the Centers for Disease Control and Prevention and the American College of Sports Medicine. JAMA. 1995;273:402-7.

27. Craig CL, Marshall AL, Sjostrom M, Bauman AE, Booth ML, Ainsworth BE, et al. International physical activity questionnaire: 12-country reliability and validity. Med Sci Sports Exerc. 2003;35:1381-95.

28. Heintjes EM, Bierma-Zeinstra SM, Berger MY, Koes BW. Lysholm scale and WOMAC index were responsive in prospective cohort of young general practice patients. J Clin Epidemiol. 2008;61:481-8.

29. Friedewald WT, Levy RI, Fredrickson DS. Estimation of the concentration of low-density lipoprotein cholesterol in plasma, without use of the preparative ultracentrifuge. Clin Chem. 1972;18:499-502.

30. Ding C, Garnero P, Cicuttini F, Scott F, Cooley H, Jones G. Knee cartilage defects: association with early radiographic osteoarthritis, decreased cartilage volume, increased joint surface area and type II collagen breakdown. Osteoarthritis Cartilage. 2005;13:198-205.

31. Peterfy CG, Guermazi A, Zaim S, Tirman PF, Miaux Y, White D, et al. WholeOrgan Magnetic Resonance Imaging Score (WORMS) of the knee in osteoarthritis. Osteoarthritis Cartilage. 2004;12:177-90.

32. Berthiaume MJ, Raynauld JP, Martel-Pelletier J, Labonte F, Beaudoin G, Bloch DA, et al. Meniscal tear and extrusion are strongly associated with 
progression of symptomatic knee osteoarthritis as assessed by quantitative magnetic resonance imaging. Ann Rheum Dis. 2005;64:556-63.

33. Guymer E, Baranyay F, Wluka AE, Hanna F, Bell RJ, Davis SR, et al. A study of the prevalence and associations of subchondral bone marrow lesions in the knees of healthy, middle-aged women. Osteoarthritis Cartilage. 2007;15:1437-42.

34. Wluka AE, Wang Y, Davies-Tuck M, English DR, Giles GG, Cicuttini FM. Bone marrow lesions predict progression of cartilage defects and loss of cartilage volume in healthy middle-aged adults without knee pain over 2 yrs. Rheumatology (Oxford). 2008;47:1392-6.

35. Wluka AE, Hanna F, Davies-Tuck M, Wang Y, Bell RJ, Davis SR, et al. Bone marrow lesions predict increase in knee cartilage defects and loss of cartilage volume in middle-aged women without knee pain over 2 years. Ann Rheum Dis. 2009;68:850-5.

36. Zanetti M, Bruder E, Romero J, Hodler J. Bone marrow edema pattern in osteoarthritic knees: correlation between MR imaging and histologic findings. Radiology. 2000;215:835-40.

37. Baranyay FJ, Wang Y, Wluka AE, English DR, Giles GG, Sullivan RO, et al. Association of bone marrow lesions with knee structures and risk factors for bone marrow lesions in the knees of clinically healthy, community-based adults. Semin Arthritis Rheum. 2007;37:112-8.

38. Khan HI, Aitken D, Blizzard L, Ding C, Pelletier JP, Pelletier JM, et al. History of knee injury and MRI-assessed knee structures in middle- and older-aged adults: a cross-sectional study. Clin Rheumatol. 2015;34(8):1463-72.

39. Berry PA, Wluka AE, Davies-Tuck ML, Wang Y, Strauss BJ, Dixon JB, et al. The relationship between body composition and structural changes at the knee. Rheumatology (Oxford). 2010;49:2362-9.

40. Lim YZ, Wang Y, Wluka AE, Davies-Tuck ML, Hanna F, Urquhart DM, et al. Association of obesity and systemic factors with bone marrow lesions at the knee: a systematic review. Semin Arthritis Rheum. 2014;43:600-12.

41. Stehling C, Liebl H, Krug R, Lane NE, Nevitt MC, Lynch J, et al. Patellar cartilage: T2 values and morphologic abnormalities at 3.0-T MR imaging in relation to physical activity in asymptomatic subjects from the osteoarthritis initiative. Radiology. 2010;254:509-20.

42. Felson DT, Niu J, Clancy M, Sack B, Aliabadi P, Zhang Y. Effect of recreational physical activities on the development of knee osteoarthritis in older adults of different weights: the Framingham Study. Arthritis Rheum. 2007;57:6-12.

43. Wang $Y$, Novera D, Wluka AE, Fairley J, Giles GG, O'Sullivan R, et al. Associations between popliteal artery wall thickness and knee structure in adults without clinical knee disease: a prospective cohort study. Arthritis Rheumatol. 2015;67:414-22.

44. Triantaphyllidou IE, Kalyvioti E, Karavia E, Lilis I, Kypreos KE, Papachristou DJ. Perturbations in the HDL metabolic pathway predispose to the development of osteoarthritis in mice following long-term exposure to western-type diet. Osteoarthritis Cartilage. 2013;21:322-30.

45. Fortier LA, Nixon AJ. Distributional changes in substance $P$ nociceptive fiber patterns in naturally osteoarthritic articulations. J Rheumatol. 1997;24:524-30.

46. Zhai G, Blizzard L, Srikanth V, Ding C, Cooley H, Cicuttini F, et al. Correlates of knee pain in older adults: Tasmanian Older Adult Cohort Study. Arthritis Rheum. 2006;55:264-71.

47. Lo GH, McAlindon TE, Niu J, Zhang Y, Beals C, Dabrowski C, et al. Bone marrow lesions and joint effusion are strongly and independently associated with weight-bearing pain in knee osteoarthritis: data from the osteoarthritis initiative. Osteoarthritis Cartilage. 2009;17:1562-9.

48. Kim IJ, Kim DH, Jung JY, Song YW, Guermazi A, Crema MD, et al. Association between bone marrow lesions detected by magnetic resonance imaging and knee pain in community residents in Korea. Osteoarthritis Cartilage. 2013:21:1207-13.

49. Sowers MF, Hayes C, Jamadar D, Capul D, Lachance L, Jannausch M, et al. Magnetic resonance-detected subchondral bone marrow and cartilage defect characteristics associated with pain and X-ray-defined knee osteoarthritis. Osteoarthritis Cartilage. 2003;11:387-93.

50. Hayes CW, Jamadar DA, Welch GW, Jannausch ML, Lachance LL, Capul DC, et al. Osteoarthritis of the knee: comparison of MR imaging findings with radiographic severity measurements and pain in middle-aged women. Radiology. 2005;237:998-1007.

51. Yusuf E, Kortekaas MC, Watt I, Huizinga TW, Kloppenburg M. Do knee abnormalities visualised on MRI explain knee pain in knee osteoarthritis? A systematic review. Ann Rheum Dis. 2011;70:60-7.
52. Hunter DJ, Gerstenfeld L, Bishop G, Davis AD, Mason ZD, Einhorn TA, et al. Bone marrow lesions from osteoarthritis knees are characterized by sclerotic bone that is less well mineralized. Arthritis Res Ther. 2009;11:R11.

53. Driban JB, Tassinari A, Lo GH, Price LL, Schneider E, Lynch JA, et al. Bone marrow lesions are associated with altered trabecular morphometry. Osteoarthritis Cartilage. 2012;20:1519-26.

54. Roemer FW, Guermazi A, Javaid MK, Lynch JA, Niu J, Zhang Y, et al. Change in MRI-detected subchondral bone marrow lesions is associated with cartilage loss: the MOST Study. A longitudinal multicentre study of knee osteoarthritis. Ann Rheum Dis. 2009;68:1461-5.

55. Lo GH, Hunter DJ, Nevitt M, Lynch J, McAlindon TE. Strong association of MRI meniscal derangement and bone marrow lesions in knee osteoarthritis: data from the osteoarthritis initiative. Osteoarthritis Cartilage. 2009:17:743-7.

56. Englund M, Guermazi A, Roemer FW, Yang M, Zhang Y, Nevitt MC, et al. Meniscal pathology on MRI increases the risk for both incident and enlarging subchondral bone marrow lesions of the knee: the MOST Study. Ann Rheum Dis. 2010;69:1796-802.

\section{Submit your next manuscript to BioMed Central and we will help you at every step:}

- We accept pre-submission inquiries

- Our selector tool helps you to find the most relevant journal

- We provide round the clock customer support

- Convenient online submission

- Thorough peer review

- Inclusion in PubMed and all major indexing services

- Maximum visibility for your research

Submit your manuscript at www.biomedcentral.com/submit
C Biomed Central 